\title{
Aleitamento materno: fatores de influência na sua decisão e duração
}

\author{
Factors influencing breastfeeding decision and duration
}

Francisca Teresa Veneziano FALEIROS

Ercília Maria Carone TREZZA²

Luana CARANDINA ${ }^{1}$

RE S U M O

Os autores analisaram diferentes fatores que podem influenciar na decisão das mães de amamentar, na duração da amamentação e as principais razões do desmame. Foram selecionados artigos publicados entre 1990 e 2004 das bases de dados Lilacs, Medline, Scielo, Bireme, utilizando as palavras-chave: desmame e fatores de risco, bem como suas versões em inglês. Alguns fatores, como maternidade precoce, baixo nível educacional e socioeconômico maternos, paridade, atenção do profissional de saúde nas consultas de pré-natal, necessidade de trabalhar fora do lar, são freqüentemente considerados como determinantes do desmame precoce. Contudo, outros, como o apoio familiar, condições adequadas no local de trabalho e uma experiência prévia positiva, parecem ser parâmetros favoráveis à decisão materna pela amamentação. Apesar da relevância dos fatores mencionados acima, os aspectos culturais e a história de vida da mãe foram os mais importantes na decisão materna pelo aleitamento e pelo momento do desmame.

Termos de indexação: aleitamento materno; desmame; fatores de risco.

\section{A B S T R A C T}

The authors analyzed different factors than can influence the mother's decision to breastfeed, the duration of breastfeeding and the main weaning reasons. Articles published from 1990 to 2004 in the databases Lilacs, Medline, Scielo and Bireme were selected, using the key-words weaning and risk factors. Some factors, such as early maternity, low maternal education and socioeconomic class, parity, attention of health professionals in prenatal care and the need to work are frequently considered determinants of early weaning. However, other factors such as family support, favorable workplace conditions and a positive previous breastfeeding experience seem to favor the mother's decision to breastfeed. Despite the relevance of the factors mentioned above, cultural aspects and the mother's life history are the most important factors determining the decision to breastfeed and the time to wean.

Indexing terms: breast feeding; weaning; risk factors.

\footnotetext{
1 Departamento de Pediatria, Faculdade de Medicina de Botucatu, Universidade Estadual Paulista. Distrito de Rubião Júnior s/n, 18618-970, Botucatu, SP, Brasil. Correspondência para/Correspondence to: F.T.V. FALEIROS.

2 Departamento de Saúde Pública, Faculdade de Medicina de Botucatu, Universidade Estadual Paulista. Botucatu, SP, Brasil.
} 


\section{N T R O D U ÇÃ O}

O aleitamento materno depende de fatores que podem influir positiva ou negativamente no seu sucesso. Entre eles, alguns relacionam-se à mãe, como as características de sua personalidade e sua atitude frente à situação de amamentar, outros referem-se à criança e ao ambiente, como, por exemplo, as suas condições de nascimento e o período pós-parto havendo, também, fatores circunstanciais, como o trabalho materno e as condições habituais de vida.

A relevância do assunto justifica-se pelas recomendações da Organização Mundial de Saúde acerca do aleitamento materno prosseguir até o sexto mês e ser complementado até os 2 anos de idade ${ }^{1}$. Diante dessa recomendação, buscou-se verificar até que ponto a mesma seria viável em nosso País bem como em países mais desenvolvidos. Pesquisas brasileiras mostram que as mães que obtiveram maior sucesso no aleitamento eram as mais velhas, mais instruídas, casadas, com experiência anterior positiva com o aleitamento e conseqüente motivação maior, com boa orientação pré-natal e apoio de outras pessoas para o manter, especialmente o do marido ${ }^{2}$.

Este trabalho pretende analisar os principais fatores que influenciam as decisões das mães de amamentar, a duração da amamentação e as principais razões do desmame. Foram selecionados artigos publicados entre 1990 a 2004, a partir das bases de dados: Lilacs, Medline, Scielo e Bireme, publicações de organismos nacionais e internacionais, dissertações e teses, utilizando os termos weaning and risk factors.

Os textos analisados neste artigo apontam como fatores de influência os seguintes.

\section{Idade materna}

Alguns autores relacionam a idade materna mais jovem à menor duração do aleitamento, talvez motivada por algumas dificuldades, como, por exemplo, um nível educacional mais baixo, poder aquisitivo menor e, muitas vezes, o fato de serem solteiras. As adolescentes, por sua vez, aliam muitas vezes sua própria insegurança e falta de confiança em si mesmas para prover a alimentação para o seu bebê, à falta de apoio das próprias mães ou familiares mais próximos, ao egocentrismo próprio dessa idade e aos problemas com a auto-imagem, alcançando, freqüentemente, um menor índice de aleitamento ${ }^{3,4}$. Em um estudo norte-americano, verificou-se que, nas mães adolescentes, a produção de leite era menor, com altas concentrações de sódio, principalmente nas primeiras 6 a 18 semanas após o parto, e que nessas mães o número de mamadas durante o dia era menor havendo, com mais freqüência, o uso de fórmulas lácteas para os seus bebês 5 . 0 número de consultas de pré-natal nesse grupo, no Brasil, costuma ser menor podendo não atingir, sequer, o número mínimo preconizado pelo Ministério da Saúde, que é de cinco consultas por gestação ${ }^{6}$.

Enquanto alguns autores consideram não haver uma associação significativa entre a idade materna e a duração do aleitamento materno outros, por sua vez, verificam que os filhos daquelas mães com mais idade mamam por mais tempo, exclusiva ou parcialmente, em relação aos filhos das mães mais jovens, especialmente quando estas tinham maior número de filhos e/ou história pregressa de sucesso em aleitamento materno ${ }^{7-10}$.

\section{Situação socioeconômica, grau de instrução e condições de trabalho maternos}

No que se refere ao grau de instrução materna, muitos estudos têm demonstrado que esse fator afeta a motivação para o amamentar. Em muitos países desenvolvidos, mães com maior grau de instrução tendem a amamentar por mais tempo, talvez pela possibilidade de um maior acesso a informações sobre as vantagens do aleitamento materno. Já em países em desenvolvimento, as mães de classes menos favorecidas, também menos instruídas, freqüentemente não casadas, começam o pré-natal mais tarde e, con- 
seqüentemente, se preocupam em decidir sobre a forma do aleitamento também mais tarde 2,10.

No Brasil, as mulheres de baixa renda foram as que menos procuraram os serviços de pré-natal e que tiveram um menor número de consultas, além de iniciá-lo mais tardiamente, resultando num menor índice de aleitamento materno entre elas. A partir do sexto mês a prevalência do aleitamento materno se inverte, sendo maior entre as mais pobres, fato talvez explicado por razões de dificuldades econômicas, que impedem a complementação com outros alimentos ou, até mesmo, com outros tipos de leite ${ }^{11}$.

Nos países não industrializados, as mulheres de classes menos favorecidas, de baixo e médio poder aquisitivo, amamentam mais que as de melhor nível socioeconômico. Nas regiões brasileiras mais desenvolvidas, o padrão de aleitamento é semelhante ao dos países desenvolvidos, ou seja, mulheres mais instruídas, de melhor nível socioeconômico, amamentam por mais tempo ${ }^{12,13}$. Em trabalho realizado em uma área urbana no sudeste do Brasil, a maioria das mães tinha renda familiar per capita e nível de escolaridade baixos, o que lhes conferia um grau de instrução mínimo, com menores possibilidades de informações mais abrangentes sobre o assunto ${ }^{14}$.

Segundo dados do Ministério da Saúde, em 1999 a prevalência do aleitamento materno exclusivo nas diferentes regiões do Brasil, até o quinto mês, era maior na Região Sul, seguida das regiões Nordeste, Norte, Centro-Oeste e Sudeste, respectivamente, variando de $64,3 \%$ a $42,8 \%$ no primeiro mês, até $19,6 \%$ a $12,15 \%$ no quinto mês. No sexto mês houve discreto aumento na prevalência do aleitamento materno exclusivo na Região Sudeste $(8,4 \%)$, em relação à Região Centro-Oeste $(7,9 \%)$. Ao analisar a prevalência do aleitamento materno exclusivo no primeiro semestre de vida, no Brasil como um todo, verificou-se que a mesma variou de $53,1 \%$ no primeiro mês, até $9,7 \%$ no sexto mês, porcentagens ainda aquém da recomendação oficial da Organização Mundial da Saúde ${ }^{15,16}$.

Em São Paulo, num grupo de mulheres trabalhadoras formais, observou-se um índice de aleitamento exclusivo três vezes maior naquelas com mais de oito anos de escolaridade, comparativamente àquelas com menos de oito anos de escolaridade. Em relação ao número de mamadas por dia, as mulheres com maior grau de instrução amamentam mais freqüentemente seus filhos no período de 24 horas $^{12,17}$.

Em relação ao trabalho materno, de modo geral, o mesmo não se apresenta como empecilho específico ao aleitamento, porque a maioria das mães não trabalha fora ou deixa de fazê-lo após o nascimento de seus bebês. Por outro lado, alguns autores referem que o trabalho materno só não é empecilho se houver condições favoráveis à manutenção do aleitamento, como, por exemplo, respeito à licença gestante, creche ou condições para o aleitamento no local e horário do trabalho. Independentemente da ocupação da mãe, o que parece ter mais importância é o número de horas trabalhadas, sendo maiores os índices de desmame quando o mesmo excede a 20 horas semanais. Vários estudos mostram que também é importante o fato de essa mãe ter ou não uma jornada dupla de trabalho, ou seja, se ocupar de todos os afazeres domésticos, além daqueles que seu trabalho fora do lar Ihe solicita. Nesse caso, mais freqüentemente, parece ocorrer o desmame ${ }^{18}$.

Silva ${ }^{19}$ verificou que a falta de apoio nas instituições e de condições ambientais para a ordenha do leite, bem como a falta de berçários, permitindo a proximidade mãe-criança, foram as principais dificuldades apontadas pelas mães em relação à manutenção da amamentação. Em São Paulo, SP, um estudo com 76 mães funcionárias de 13 indústrias, mostrou que $97 \%$ delas iniciaram o aleitamento materno, mas, apesar de 55\% ainda amamentarem após o quarto e quinto mês do nascimento, quando da sua volta ao trabalho, apenas $12 \%$ o faziam exclusivamente ${ }^{20}$. 
Os planos de retorno ao trabalho, segundo alguns autores, não parecem interferir com a decisão de iniciar o aleitamento, porém, se esse retorno ocorre já nos primeiros dois a três meses após o parto, isso parece dificultar o seu sucesso. Muitas vezes, essa volta precoce ao trabalho resulta de pressões, principalmente no caso das mães não registradas, pelo medo de perder seus empregos ${ }^{21,22}$. Observou-se, também, que a maioria das mães desconhecia seus direitos trabalhistas ou conhecia muito pouco sobre o assunto ${ }^{23}$.

\section{Situação conjugal, o papel do pai e de outras pessoas significantes para a mãe}

O fato de as mães terem uma união estável e o apoio de outras pessoas, especialmente do marido ou companheiro, parece exercer uma influência positiva na duração do aleitamento materno. Tanto o apoio social e econômico, como o emocional e o educacional parecem ser muito importantes, sendo o companheiro a pessoa de maior peso nesses diferentes tipos de apoio ${ }^{24}$.

Em trabalhos realizados no Texas e em Cleveland, EEUU, que analisam o papel do pai frente ao aleitamento materno, geralmente se considera apenas sua atitude em relação ao assunto, sem maior caracterização dos indivíduos. A atitude positiva do pai parece exercer um maior efeito na motivação e na capacidade da mãe para amamentar. Num grupo de crianças cujos pais eram bastante favoráveis ao aleitamento, verificou-se que 75,0\% eram aleitadas exclusivamente e $98,0 \%$ delas pelo menos parcialmente. Comparando-as com crianças cujos pais eram indiferentes ou desfavoráveis, a taxa de aleitamento materno exclusivo caiu para $7,7 \%$. Noutro grupo de pais favoráveis ao aleitamento materno, a maioria acreditava ser ele o melhor para o bebê, mas, naqueles mais propensos ao aleitamento artificial, muitos pensavam ser ruim para as mamas e um fator de interferência na relação sexual do casal $^{25,26}$. Apesar desses trabalhos serem norte-americanos, verifica-se, na prática, que essa idéia, mesmo que não tão difundida hoje, ainda está presente para algumas mães com diferentes conceitos e tabus a respeito da amamentação. Analisando esses estudos, pode-se concluir que seria importante aumentar a informação aos pais sobre as vantagens do aleitamento materno e do seu real significado, iniciando-se esse processo educativo já na infância e adolescência. Isso ajudaria não só os pais a optarem mais pelo aleitamento materno, como também a manejar melhor a nova situação do casal promovendo, inclusive, satisfação e sucesso no aleitamento.

\section{Paridade materna, experiência anterior e intenção de amamentar}

A influência da paridade materna na decisão pelo tipo de aleitamento é um fator bastante discutível na literatura, com alguns estudos sugerindo que as primíparas, ao mesmo tempo que mais propensas a iniciar o aleitamento, costumam mantê-lo por menos tempo, introduzindo mais precocemente os alimentos complementares, parecendo haver para as multíparas uma forte correlação entre o modo como seus filhos anteriores foram amamentados e como este último o será10,27. Observou-se também que as mães desmamavam mais precocemente os primogênitos e mantinham o aleitamento materno tanto mais prolongado quanto maior o número de ordem da criança na família. A razão estaria, talvez, relacionada à insegurança da "mãe de primeira viagem", eventualmente mais jovem, com menor grau de instrução e menor experiência de vida ${ }^{21}$. Em se tratando de ter ou não uma experiência anterior com aleitamento materno, as mães que tiveram uma experiência prévia positiva, provavelmente, terão mais facilidade para estabelecê-lo com os demais filhos. Parece haver uma forte correlação entre a intenção da gestante em amamentar seu filho e a duração da amamentação ${ }^{28}$. Donath ${ }^{29}$ encontrou, numa coorte de 1400 gestantes, esse fato como o melhor preditor para o aleitamento materno adequado, sendo melhor que outros fatores demográficos combinados. O fato de a mãe, muitas vezes, falhar na amamentação, apesar de um forte desejo de efetivá-la, pode ser devido à falta de acesso à orientação e ao apoio adequado de profissionais 
ou de pessoas mais experientes dentro ou fora de sua família ${ }^{30,31}$.

Considerando que cada nascimento se dá em contextos não necessariamente iguais, ou seja, diferenças de idade, de condições socioeconômicas ou de situação conjugal da mãe, o simples fato de ter uma experiência prévia, talvez não seja suficiente como estímulo para amamentação dos filhos subseqüentes. Portanto, a dificuldade de análise da influência dessa variável se deve a múltiplos fatores e às mudanças na dinâmica familiar ocorridas com o passar do tempo.

\section{O papel do profissional de saúde}

Pesquisas mostram que, embora a maioria dos pré-natalistas aconselhe o aleitamento materno às mães que ainda não se decidiram, poucos falam sobre o assunto no primeiro trimestre e muitos recomendam a complementação com fórmulas lácteas ${ }^{23}$. Com isso, infelizmente, o próprio profissional de saúde, entre eles o médico, pode contribuir com suas próprias atitudes para o desmame precoce $\mathrm{e}^{22,32}$.

Em 1981, segundo dados da Secretaria de Saúde de São Paulo, cerca de 13\% das mulheres não tinham acesso ao pré-natal e, quando o tinham, a média de consultas por gestação era de 2,95, número muito aquém do preconizado pelo Ministério da Saúde, ou seja, mínimo de 5 consultas, com intervalo não superior a 8 semanas entre elas $^{33}$. Segundo o Sistema de Informações sobre Nascimentos (SINASC), em 2001 a situação se apresentava bem melhor, sendo que nas diferentes regiões brasileiras, houve um predomínio de 7 ou mais consultas de pré-natal nas Regiões Centro-Oeste, Sul e Sudeste, em ordem crescente e de 4 a 6 consultas nas Regiões Norte e Nordeste, respectivamente ${ }^{34}$. Embora hoje a situação esteja bem melhor em todas as Regiões do Brasil, ainda não se nota um incremento na prevalência do aleitamento materno correspondente à meta desejada.

Mais importante que o início precoce e a freqüência às consultas de pré-natal são as atitudes dos profissionais, consideradas como indicadores indiretos da qualidade da assistência prestada. Um dado interessante parece ser a experiência pessoal do próprio médico ou de seu cônjuge com aleitamento materno o que possibilitaria uma abordagem mais consistente do assunto por eles com suas pacientes ${ }^{23,35}$.

Apesar das deficiências no conhecimento sobre o assunto, tanto residentes como médicos mais experientes parecem fortemente convencidos do seu papel na promoção do aleitamento. A maioria acredita que o conselho do médico e de outros profissionais de saúde é um meio muito importante de aumentar as taxas de aleitamento materno, e refere que o desconhecimento de seus benefícios e das estratégias para a sua orientação se deve às poucas oportunidades de empregar as habilidades de aconselhamento durante os anos de formação. Santiago et al. ${ }^{35}$ demonstraram que o grupo de pediatras com treinamento específico em aleitamento materno se equiparou às equipes multidisciplinares encarregadas dessas mesmas orientações. Obviamente, aquele profissional que tiver um embasamento teórico e técnico insuficiente terá maiores dificuldades na abordagem do assunto ${ }^{36}$

\section{Rotinas hospitalares}

Quanto ao tipo de parto, parece haver maior facilitação para o estabelecimento da lactação mais precoce e efetiva no parto vaginal, uma vez que não há o fator dor incisional ou o efeito pós-anestésico da cesárea, dificultando, portanto, as primeiras mamadas. No parto normal, o primeiro contato mãe-filho ocorre mais precocemente, enquanto que na cesárea, dificilmente a criança vai até a mãe antes das primeiras seis horas pós-parto, propiciando a introdução de fórmula láctea ou glicose para o recém-nascido já no berçário e, o que é pior, em mamadeira ${ }^{37,38}$.

No Brasil, em 1996, a Sociedade Civil do Bem-Estar Familiar (BEMFAM) mostrou que o parto cesárea prevaleceu em 36\% dos casos, chegando a 52 \% no Estado de São Paulo e a $49 \%$ na região Centro-Oeste, com os menores porcentuais nas 
regiões Nordeste e Norte, 20\% e 25\%, respectivamente $^{39}$. Esses índices representam o dobro do padrão internacional recomendado pela Organização Mundial de Saúde, que é de 10\% a 15\% de cesarianas. Essa recomendação se fundamenta nos riscos indicativos da interferência cirúrgica, que pode afetar a saúde da mulher no puerpério, no seu futuro obstétrico e no sucesso do aleitamento materno. É interessante notar que apenas 10\% das cesáreas foram indicadas por não progressão do trabalho de parto, podendo significar que grande número delas são eletivas e, provavelmente, desnecessárias. A escolaridade da mulher também se revelou fortemente associada à ocorrência de parto cesárea, predominado esse tipo de parto nas que tinham o maior número de anos de estudo.

Hoje, segundo informações do Anuário Estatístico de Saúde do Brasil, em 2001, a ocorrência de partos cesárea ainda ultrapassa essas recomendações, ocorrendo cerca de $38,00 \%$ de partos cesárea e $50,75 \%$ de partos normais em todo o Brasil. Se considerarmos as diferentes Regiões, a incidência de cesáreas varia de 25,65\% na Região Nordeste para 45,95\% na Região Sudeste, ocorrendo em menores proporções nas Regiões Norte e Nordeste ${ }^{34}$.

Num estudo feito em Pelotas, RS, em 1998, a duração da amamentação foi similar entre os nascidos por parto vaginal ou cesárea emergencial, porém, as crianças nascidas por cesárea eletiva apresentaram um risco três vezes maior de interrupção da lactação já no primeiro mês de vida ${ }^{40}$.

O sistema de alojamento conjunto tem como objetivo principal fortalecer o vínculo mãe-filho e estimular a prática do aleitamento materno, porém, mesmo nos hospitais que têm esse sistema, freqüentemente, o recém-nascido, antes de ser colocado junto à sua mãe, é levado ao berçário para os primeiros cuidados, prolongando, assim, o intervalo entre o parto e a primeira mamada, dificultando o estabelecimento precoce do vínculo mãe-filho e da lactação. Um outro fator sabidamente promotor de maior duração do aleitamento materno é o regime de livre-demanda, para o qual esse sistema atua como um grande facilitador $8,41,42$.

Num trabalho realizado no México, a freqüência do aleitamento materno exclusivo foi maior em mães que ficaram em alojamento conjunto após o parto, influenciada também pelo tipo de parto, enquanto que o aleitamento misto foi maior nas que não ficaram no alojamento conjunto, independentemente do tipo de parto. Concluiu-se, portanto, que o alojamento conjunto interfere positivamente na amamentação, mesmo nas mães que tiveram parto cesárea, pois favorece um maior contato mãe-filho ${ }^{43}$.

\section{Problemas com o aleitamento}

A literatura aponta que as mães têm, geralmente, noção das vantagens do aleitamento materno e referem doenças maternas ou da criança e o trabalho fora do lar como problemas pouco freqüentes em relação à manutenção do mesmo. No entanto, apontam como relevantes os problemas relacionados à "falta de leite", "leite fraco", problemas mamários e a recusa do bebê em pegar o peito. Essas razões, apontadas mais freqüentemente, talvez se devam ao fato de a mulher atual ter uma vivência mais ansiosa e tensa e possivelmente, à falta de um suporte cultural que havia nas sociedades tradicionais, nas quais as avós transmitiam às mães informações e um treinamento das mesmas em relação ao aleitamento, incentivando-as para tal. Outra razão de preocupação, atualmente, poderiam ser as cirurgias de redução ou próteses mamárias ${ }^{23,44}$.

\section{CONSIDERAÇÕES FINAIS}

Embora muitos dos fatores citados pareçam explicar as causas do desmame precoce, é possível sugerir outras razões que o expliquem, ligadas ao ambiente, à personalidade materna, às suas emoções, à relação com o marido e a família, às influências culturais e à sua resposta aos diferentes problemas do cotidiano. É relevante também o 
papel dos órgãos de comunicação e da indústria de alimentos infantis.

Muitas vezes, os serviços e os profissionais de saúde enfatizam o aspecto biológico da amamentação, em detrimento de questões singulares da mulher, que podem incluir tanto emoções positivas quanto negativas em relação ao ato de amamentar.

Portanto, é de fundamental importância que não se generalize a capacidade de amamentar, sem que antes se considerem as variáveis contextuais. Para que a mulher possa assumir com mais segurança o papel de mãe e de provedora do alimento de seu filho, ela precisa se sentir adequadamente assistida nas suas dúvidas e dificuldades.

Cabe, principalmente, aos profissionais de saúde a tarefa de garantir, a cada mãe, uma escuta ativa, ou seja, de saber ouvi-la, dirimir suas dúvidas, entendê-la e esclarecê-la sobre suas crenças e tabus, de modo a tornar a amamentação um ato de prazer e não o contrário.

\section{REFERÊ N CIAS}

1. Brasil. Ministério da Saúde. Instituto Nacional de Alimentação e Nutrição. Programa Nacional de Incentivo ao Aleitamento Materno. Proteção, Promoção e Apoio ao Aleitamento Materno: o papel especial dos serviços materno-infantis. Genebra: OMS; 1989.

2. Escobar AMU, Ogawa AR, Hiratsoka M, Kawashita MY, Teruya PY, Grisi S, et al. Aleitamento materno e condições sócio-econômico-culturais: fatores que levam ao desmame precoce. Rev Bras Saúde Mater Infant. 2002; 2(2):253-61.

3. Peterson CE, Da-Vanzo J. Why are teenagers in the United States less likely to breast-feed than older women? Demography. 1992; 29(3):431-50.

4. Gigante DP, Victora CG, Barros FC. Nutrição materna e duração da amamentação em uma coorte de nascimento de Pelotas, RS. Rev Saúde Pública. 2000; 34(3):259-65.

5. Motil KJ, Kertz B, Thotathuchery M. Lactational performance of adolescent mothers shows preliminary differences from that of adult women. J Adolesc Health. 1997; 20(6):442-9.

6. São Paulo (Estado). Secretaria do Estado de Saúde. Uma resolução na área da saúde. A experiência do sistema unificado e descentralizado da Saúde do Estado de São Paulo. São Paulo: IMESP; 1988.

7. Lima TM, Osório MM. Perfil e fatores associados ao aleitamento materno em crianças menores de 25 meses, da Região Nordeste do Brasil. Rev Bras Saúde Mater Infant. 2003; 3(3):305-14.

8. Vieira GO, Almeida JAG, Silva LR, Cabral VA, Netto PVS. Fatores associados ao aleitamento materno e desmame em Feira de Santana, Bahia. Rev Bras Saúde Mater Infant. 2004; 4(2):143-50.

9. Vieira MLF, Silva JLC Pinto, Barros Filho AA. A amamentação e a alimentação complementar de filhos de mães adolescentes são diferentes das de filhos de mães adultas? J Pediatr. 2003; 79(4): 317-24

10. Venâncio SI, Escuder MM, Kitoko P, Réa MF, Monteiro CA. Freqüência e determinantes do aleitamento materno em municípios do Estado de São Paulo. Rev Saúde Pública. 2002; 36(3):313-8.

11. Kummer SC, Giugliani ERJ, Susin LO, Folleto JL, Lermen NR, Wu VYJ, et al. Evolução do padrão de aleitamento materno. Rev Paul Saúde Pública. 2000; 34(2):143-8.

12. Réa MF, Berquó ES. Impact of the Brazilian national breast-feeding programme on mothers in greater São Paulo. Bull World Health Organ. 1990; 68(3): 365-71.

13. Giugliani ERJ, Issler RMS, Kreutz G, Meneses C, Justo EB, Kreutz $V$, et al. Breastfeeding pattern in a population with different levels of poverty in Southern Brazil. Acta Paediatr. 1996; 85(12): 1499-500.

14. Pedroso GC, Puccini RF, Silva EMK, Silva NN, Alves MCGP. Prevalência de aleitamento materno e introdução precoce de suplementos alimentares em área urbana do Sudeste do Brasil, Embu, SP. Rev Bras Saúde Mater Infant. 2004; 4(1):45-58.

15. Brasil. Ministério da Saúde. Prevalência de aleitamento materno exclusivo nas diferentes regiões do Brasil [Internet]. Brasília; 2004 [acesso em 19 ago 2004]. Disponível em: http://portal. saude.gov.br/saude/aplicacoes/anuario2001

16. World Health Organization. The World Health Organization's infant-feeding recommendation. Bull World Health Organ. 1995; 73(4):165-74.

17. Bueno MB, Souza JMP, Souza SB, Paz SMRS, Gimeno SGA, Siqueira AAF. Riscos associados ao processo de desmame entre crianças nascidas em hospital universitário de São Paulo, entre 1998 e 1999: estudo de coorte prospectivo do primeiro ano de vida. Cad Saúde Pública. 2003; 19(5): 1456-60.

18. Gielen AC, Faden RR, O'Campo P, Brown H, Paige DM. Maternal employment during the early 
postpartum period: effects on initiation and continuation of breast-feeding. Pediatrics. 1991; 87(3):298-305.

19. Silva IA. Situação de amamentação entre mulheres trabalhadoras e alunas de graduação e pós-graduação de uma universidade pública. Acta Scient Cienc Saúde. 2003; 25(2):215-25.

20. Réa MF, Venâncio SI, Batista LE, Gomes dos Santos $R$, Greiner T. Possibilidades e limitações da amamentação entre mulheres trabalhadoras formais. Rev Saúde Pública. 1997; 31(2):149-56.

21. Hammer LD, Bryson S, Agras S. Development of feeding practices during the first 5 years of life. Arch Pediatr Adolesc Med. 1999; 153(2):189-94.

22. Ciaccia MCC, Ramos JLA, Issler H. Amamentação e trabalho da mulher: como conciliar. Rev Paul Pediatr. 2003; 21(3):83-8.

23. Giugliani, ERJ. O aleitamento materno na prática clínica. J Pediatr. 2000; 76 (3 Supl.):S238-52.

24. Giugliani ERJ 1994. Amamentação: como e por que promover. J Pediatr. 1994; 70(3):138-51.

25. Freed GL, Fraley JK, Schanler RJ. Attitudes of expectant fathers regarding breast-feeding. Pediatrics. 1992; 90(2 Pt1):224-27.

26. Littman H, Mebendorp SV, Goldfarb J. The decision to breastfeed: the importance of father's approval. Clin Pediatr. 1994; 33(4):214-9.

27. Berra S, Sabolsky J, Rajmil L, Passamonte R, Pronsato $J$, Botinof M. Correlates of breastfeeding duration in an urban cohort from Argentina. Acta Paediatr. 2003; 92(8):952-7.

28. Vogel AM. Intended plans for breastfeeding duration: a simple tool to predict breastfeeding outcome. Acta Paediatr. 2003; 92(3):270-1.

29. Donath SM, Amir LH, Alspac Study Team. Relationship between prenatal infant feeding intention and initiation and duration of breastfeeding: a cohort study. Acta Paediatr. 2003; 92(3):352-6.

30. Silva HML. O estudo do aleitamento materno a partir do olhar da mulher: a (des)mistificação da mãe biológica [dissertação]. João Pessoa: Universidade Federal da Paraíba; 1999.

31. Nakano MAS, Mamede MV. A prática do aleitamento materno em um grupo de mulheres brasileiras: movimento de acomodação e resistência. Rev Latino Am Enf. 1999; 7(3):69-76.

32. Dearden K, Altaye M, Maza I, Oliva M, Stone-Jimenez M, Morrow A, et al. Determinants of optimal breast-feeding in peri-urban Guatemala City, Guatemala. Rev Panam Salud Publica. 2002; 12(3):185-92.
33. São Paulo (Estado). Secretaria do Estado de Saúde. Grupo de saúde da mulher. Subprograma de saúde da mulher. Subsídios e diretrizes. São Paulo: DTN; 1986.

34. Brasil. Ministério da Saúde. DATASUS. Nascimentos por residência da mãe por consulta pré-natal, segundo Região/UF, período 2001 [Internet]. Brasília; 2004. [acesso em 19 ago 2004]. Disponível em: http://tabnet.datasus.gov.br/cgi/ tabagi.exe? sinasc/cnv/nvinf.def

35. Santiago LB, Bettiol H, Barbieri MA, Gutierrez MRP, Del Ciampo LA. Incentivo ao aleitamento materno: a importância do pediatra com treinamento específico. J Pediatr. 2003; 79(6):504-12.

36. Vitollo MR, Accioly E, Moraes DEB de, Franceschini SCC. Conhecimento sobre aleitamento materno entre estudantes do último ano do curso de Medicina. Rev Ciênc Méd. (Campinas) 1998; 7(1):27-33.

37. Carvalhaes MABL, Correa CRH. Identificação de dificuldades no início do aleitamento materno mediante aplicação de protocolo. J Pediatr. (Rio de Janeiro) 2003; 79(1):13-20.

38. Figueiredo MG, Sartorelli DS, Zan TAB, Garcia E, Silva LC, Carvalho FLP, et al. Inquérito de avaliação rápida das práticas de alimentação infantil em São José do Rio Preto, SP, Brasil. Cad Saúde Pública. 2004; 20(1):172-9.

39. Sociedade Civil do Bem-Estar Familiar no Brasil. Pesquisa nacional sobre demografia e saúde. Rio de Janeiro; 1996. p.103-36.

40. Wiederpass E, Barros FC, Victora CG, Tomasi E, Halpern R. Incidência e duração da amamentação conforme o tipo de parto: estudo longitudinal no Sul do Brasil. Rev Saúde Pública. 1998; 32(3)-225-31.

41. Giugliani ERJ, Issler RMS, Justo EB, Sefrin CF, Hartman RM, Carvalho NM. Risk factors for early termination of breast-feeding in Brazil. Acta Paediatr. 1992; 81(6-7):484-7.

42. Del Ciampo LA, Ricco RG, Muccillo G, Bettiol H, Daneluzzi JC. Influências dos diferentes tipos de alojamento sobre recém-nascidos na prática do aleitamento materno. J Pediatr. 1994; 70(1):10-5.

43. Huerta SF, Silva IC. Alojamento conjunto madre-hijo y lactancia exclusiva. Salud Pública Méx. 1997; 39(2):110-6

44. Hughes $V$, Owen J. Is breast-feeding possible after breast surgery? Am J Matern Child Nurs. 1993; 18(4):213-7.

Recebido em: 25/4/2005

Versão final reapresentada em: 6/6/2006 Aprovado em: 14/8/2006 